\section{Subregistro y exhaustividad de los sistemas de vigilancia de tuberculosis en una región del Perú: un análisis de captura-recaptura}

\author{
Underreporting and exhaustiveness of \\ tuberculosis surveillance systems in a region \\ of Peru: a capture-recapture analysis
}

\section{Subnotificação e exaustividade dos sistemas de vigilância da tuberculose em uma região do Peru: uma análise de captura-recaptura}

\section{Resumen}

Esta investigación buscó determinar el subregistro de casos de tuberculosis (TB) en la Región Cajamarca, Perú, en los años 2017 y 2018, mediante el método de captura recaptura, asimismo estimar la tasa de incidencia a través de este método, evaluar la exhaustividad de los sistemas de vigilancia, y describir las características epidemiológicas de la TB en el periodo estudiado. Se realizó un estudio descriptivo, retrospectivo en la Región Cajamarca; se analizaron dos sistemas de vigilancia: el Sistema de Información Gerencial de Tuberculosis (SIGTB) y el Sistema Epidemiológico de Tuberculosis (SIEPI-TB), en base al número de casos registrados en cada sistema se aplicó el método de captura y recaptura para obtener una estimación de casos reales, validando los datos con un modelo log-lineal en el entorno estadístico $R$. Los mayores subregistros en los sistemas fueron: 40,7\% en el 2017 y 25,6\% en el 2018; se encontró que las tasas de incidencia estimadas en ambos años fueron superiores a las reportadas por los sistemas oficiales, el sistema de vigilancia más exhaustivo fue el SIEPI-TB. Los resultados indican la existencia de un preocupante subregistro $y$ la necesidad de monitoreo de los sistemas de vigilancia de tuberculosis.

Tuberculosis; Omisiones de Registro; Vigilancia Sanitaria
Hans Ramón Quiroz-Ruiz 1,2

Jorge Luis Sosa-Flores 3,4

Fiorella Nathali Hernández-Palomino 1

\author{
Correspondencia \\ H. R. Quiroz-Ruiz \\ Facultad de Ciencias Biológicas, Universidad Nacional \\ Pedro Ruiz Gallo. \\ Calle Juan XXIII 391, Ciudad Universitaria, Lambayeque, \\ 14013, Perú. \\ quirozh_rci@hotmail.com \\ 1 Facultad de Ciencias Biológicas, Universidad Nacional Pedro \\ Ruiz Gallo, Lambayeque, Perú. \\ 2 Laboratorio de Referencia Regional de Salud Pública, \\ Dirección Regional de Salud Cajamarca, Cajamarca, Perú. \\ 3 Facultad de Medicina Humana, Universidad Nacional Pedro \\ Ruiz Gallo, Lambayeque, Perú. \\ ${ }_{4}$ Hospital Nacional Almanzor Aguinaga Asenjo, Chiclayo, Perú.
}




\section{Introducción}

La tuberculosis (TB) es una enfermedad causada por la bacteria Micobacterium tuberculosis y es la principal causa de muerte por un agente infeccioso 1. En las Américas, el 87\% de los casos de TB se encuentran en diez países; sin embargo, poco más de la mitad se concentran en Brasil, Perú y México; con un estimado de 37 mil casos de TB en el Perú 2 , en este país la enfermedad ocupa el décimo quinto lugar de las causas de muerte $3 \mathrm{y}$ estudios realizados sugieren la posibilidad de subregistros de casos 4,5; sin embargo, el Perú no ha implementado ni planificado estudios de inventario nacional del subregistro de esta enfermedad 1. En Perú la notificación, entendida como el registro de casos, se realiza de manera obligatoria en dos sistemas oficiales: el Sistema Epidemiológico de Tuberculosis (SIEPI-TB), dependiente del Centro Nacional de Epidemiología, Prevención y Control de Enfermedades y el Sistema de información Gerencial de Tuberculosis (SIGTB), el cual se estableció en el año 2016 por la Ley no 30.287, este aplicativo depende de la Dirección de Prevención y Control de Tuberculosis 6; ambos sistemas registran información basada principalmente en la ficha epidemiológica y en las tarjetas de control de tratamiento de las personas afectadas con tuberculosis. En la Región Cajamarca algunos autores señalan tasas de incidencia de 13.2 casos por 100 mil habitantes 3, el SIEPI-TB reportó 15 casos por 100 mil habitantes en el año 2017 y 16 casos por 100 mil habitantes en el año 2018. En esta región no se ha evaluado previamente el subregistro, ni se ha determinado la exhaustividad de los sistemas de vigilancia de TB; por lo que se planteó como objetivos: determinar el subregistro de casos de TB en la Región Cajamarca en los años 2017 y 2018, mediante el método de captura recaptura, así como estimar la tasa de incidencia a través de este método, evaluar la exhaustividad de los sistemas de vigilancia y describir las características epidemiológicas de la TB en el periodo estudiado.

\section{Métodos}

Esta investigación fue de tipo descriptiva, retrospectiva, durante los años 2017 y 2018, en la Región Cajamarca, Perú; en este periodo la Dirección Regional de Salud proyectó una población de 1.481685 habitantes en el 2017, y 1.529.036 habitantes en el 2018; se utilizó información de fuente secundaria, proveniente de bases de datos generadas por los dos sistemas de vigilancia de tuberculosis: el SIGTB y el SIEPI-TB; las bases fueron obtenidas con fecha de corte al 30 de octubre de 2019 y se organizaron en tablas Excel 2013 (https://products.office.com/), depurando la información según las variables de interés. Las bases se organizaron por nombres y apellidos, número de documento nacional de identidad y fecha de diagnóstico; para la deduplicación de casos, se consideró aquellos con más de un registro en una misma base de datos y que tenían la misma fecha de diagnóstico; asimismo se eliminó el primer registro de los casos que fueron informados como TB sensible y, posteriormente, como TB resistente; el linkage se realizó para la selección de casos comunes en ambas bases de datos, para lo cual se utilizó Excel 2013, siguiendo un procedimiento visual, en dos etapas: primero verificando cuántos casos con nombres completos iguales aparecen registrados simultáneamente en ambas bases y, en una segunda etapa, se verificó cuántos casos con el mismo número del documento nacional de identidad aparecen registrados en ambas bases. Las bases se guardaron siguiendo la siguiente estructura, donde 1 indica que el caso de TB aparece registrado en esa base y 0 indica que no lo está. Se aplicó el método de captura recaptura mediante el estimador propuesto por Chapman, siguiendo el modelo de dos capturas 7 :

\begin{tabular}{cccc}
\hline & \multicolumn{2}{l}{ SIEPI-TB } \\
\cline { 3 - 4 } & & $\mathbf{0}$ & $\mathbf{1}$ \\
\hline SIGTB & $\mathbf{0}$ & $a$ & $b$ \\
& $\mathbf{1}$ & $c$ & $d$ \\
\hline
\end{tabular}


Donde $a$ es el número de casos desconocidos que no fueron registrados en ningún sistema; $b$ es el número de casos solamente registrado en el SIEPI-TB; $c$ corresponde al número de casos registrados únicamente en el SIGTB, $d$ corresponde al número de casos comunes a ambas listas. El número total de casos estimados es igual a $N$, donde:

$$
N=\frac{(c+d+1)(d+b+1)}{d+1}-1
$$

La validación estadística de la estimación de casos se realizó en el entorno estadístico R, versión 3.6.1 (http://www.r-project.org); descargando dos librerías readr y Rcapture, la matriz de datos se guardó en formato de texto delimitado por tabulaciones, siguiendo la siguiente estructura como ejemplo:

\begin{tabular}{cc}
\hline SIEPI-TB & SIGTB \\
\hline 1 & 0 \\
1 & 1 \\
0 & 1 \\
\hline
\end{tabular}

La ruta de la matriz se encontró con la función $>$ file.choose(), se dio nombre a la matriz de datos y se insertó con la función genérica <- read.table(), posteriormente se cargó la librería Rcapture, la descripción estadística se obtuvo con la función $>$ desc<-descriptive(), analizando la heterogeneidad de los datos mediante la función $>$ plot(desc); finalmente mediante un modelo log-lineal con la función $>$ closedp se estimó el número real de casos; los intervalos de confianza se determinaron con la función profileCI 8,9; para la elección del modelo se consideró las desviaciones, el criterio de información de Akaike y el criterio de información bayesiano (AIC) 7,10; se eligió el modelo Mt el cual considera que las probabilidades de captura pueden variar en las diferentes sesiones.

El subregistro del sistema se determinó mediante:

$$
\text { Subregistro de un sistema } \%=100-\frac{\text { casos resgistrados } \times 100}{N}
$$

La tasa de exhaustividad fue calculada según lo indicado por Múñiz-González et al. 11.

$$
\begin{gathered}
\text { Exhaustividad del SIGTB }=\frac{(c+d) \times 100}{N} \\
\text { Exhaustividad del SIEPITB }=\frac{(b+d) \times 100}{N} \\
\text { Exhaustividad combinadas SIEPITB y SIGTB }=\frac{(c+b+d) \times 100}{N}
\end{gathered}
$$

Las tasas de incidencia se calcularon con la población anual estimada a nivel regional y con el número total de casos estimados mediante la captura-recaptura. Las características epidemiológicas estudiadas fueron edad, sexo, aseguramiento de salud, criterio diagnóstico, localización de la enfermedad, perfil de susceptibilidad, factores de riesgo, comorbilidades y condición de egreso, estas variables cualitativas se presentaron en número y porcentaje.

Consideraciones éticas. Se aseguró la confidencialidad y protección de datos de acuerdo a la ley peruana. La investigación fue aprobada por el comité de ética en investigación del Hospital Nacional Almanzor Aguinaga Asenjo (constancia de aprobación 054) y cuenta con la autorización de la Dirección Regional de Salud Cajamarca (DIRESA Cajamarca) a través de la unidad de investigación. 


\section{Resultados}

\section{Estimación del número de personas afectadas con tuberculosis}

Se calculó en número real de casos de TB mediante el estimador descrito (Tabla 1) encontrando valores muy cercanos a los estimados en R (Tabla 2). En el año 2017 se estimó 275 casos de tuberculosis en toda la Región Cajamarca (IC95\%: 262.4-289.43), mientras que en el año 2018 se estimó 277 casos de TB (IC95\%: 270.98-284.85).

\section{Subregistro y exhaustividad de los sistemas}

En el año 2017, 2 de cada 10 casos no fueron registrados en el SIEPI-TB y 4 de cada 10 casos de tuberculosis no fueron registrados en el SIGTB. En el año 2018, 1 de cada 10 casos de TB no fue registrado en SIEPI-TB y 3 de cada 10 casos no se registraron en el SIGTB (Tabla 3). Las tasas de exhaustividad se muestran en la misma tabla.

\section{Características epidemiológicas}

De la totalidad de personas afectadas con TB, el 94.1\% contaba con algún tipo de seguro de salud, además se encontró que la edad promedio de las personas afectadas con TB fue 42 años, predominando el sexo masculino con un valor cercano al 63.4\%; asimismo, el 58.8\% se diagnosticaron por criterio bacteriológico, seguido por $19 \%$ de casos diagnosticados por criterio radiológico.

Acerca de la localización de la enfermedad, el 75.2\% de casos fueron principalmente TB-pulmonar, seguida de la forma pleural; del total de casos presentados en el periodo estudiado predomina la TB sensible con un 97.4\%; se determinó que la asociación TB-diabetes estuvo presente en el 4.5\% de casos y la asociación TB-VIH en el 4.1\%; asimismo 6.9\% de casos presentó como factor asociado el alcoholismo; respecto al egreso de los casos del sistema, el 26.7\% fueron dados de alta como curados, sin embargo, se evidenció un mayor porcentaje sin condición de egreso. La Tabla 4 muestra los datos epidemiológicos del periodo estudiado.

\section{Discusión y conclusión}

Esta investigación constituye el primer estudio de este tipo en la Región Cajamarca, los datos obtenidos respecto a los subregistros concuerdan con valores reportados por la Organización Mundial de la Salud, la cual estima que hasta un $40 \%$ de casos de TB no son informados a los sistemas de vigilancia 12 , algunos países como Colombia, Inglaterra, Brasil y China reconocen subregistros similares 13,14,15; por otra parte en Perú no hay estudios de inventario nacional de subregistro de TB 1 , pero se reconoce la existencia del mismo, vinculado a comunidades indígenas 5 , situación que se presenta en la región estudiada; además de diversos factores 13 , como población rural, zonas sin telefonía y limitaciones culturales y lingüísticas.

Acerca de las tasas de incidencia, estimadas mediante el método de captura recaptura, esta investigación encontró que en el año 2017 la tasa de incidencia acumulada -estimada mediante la metodología empleada- fue de 19 casos por cada 100 mil habitantes y en el 2018 fue de 18 casos por cada 100 mil habitantes, se obtuvo valores superiores al de un estudio previo 3 y a los reportados por los sistemas de vigilancia de la región; a través del método empleado, diversos autores encontraron tasas superiores a las reportadas por los sistemas oficiales, sugiriendo que estos subestimaban los casos 4,11,16,17,18.

Es necesario indicar que a nivel nacional no se encontró estudios que permitan comparar los datos obtenidos, respecto a la exhaustividad de los sistemas de vigilancia de TB empleados en Perú. Los resultados indican que el SIEPI-TB es más exhaustivo que el SIGTB, sin embargo, el SIEPI-TB no está actualizado, en cuanto a la condición de egreso de pacientes; consideramos que ambos sistemas son complementarios. Asimismo, se indica que la exhaustividad de los sistemas de vigilancia en salud dependerá de la realidad estudiada, como muestran distintos autores 11,16. Los hallazgos obtenidos en este estudio indican que esta metodología es útil para determinar la exhaustividad o integridad de la vigilancia de TB 18 . 
Tabla 1

Casos de tuberculosis (TB) registrados y estimados en la Región Cajamarca, Perú, años 2017 y 2018.

\begin{tabular}{lcccc}
\hline Año & SIEPI-TB & SIGTB & Casos comunes * & $\mathbf{n}$ \\
\hline 2017 & 219 & 163 & 130 & 275 \\
2018 & 245 & 206 & 182 & 277 \\
\hline
\end{tabular}

SIEPI-TB: Sistema Epidemiológico de Tuberculosis; SIGTB: Sistema de Información Gerencial de Tuberculosis.

Fuente: elaboración propia en base a registros deduplicados de los sistemas de vigilancia y aproximación de casos mediante el estimador de Chapman.

* Casos comunes: casos registrados en ambos sistemas a la vez.

Tabla 2

Casos de tuberculosis (TB) estimados en entorno estadístico R, se muestra tres modelos para el linkage Sistema Epidemiológico de Tuberculosis/Sistema de Información Gerencial de Tuberculosis (SIEPI-TB/SIGTB), años 2017 y 2018.

\begin{tabular}{ccccccc}
\hline Año/Modelo & NR & SE & DM & GL & AIC & BIC \\
\hline 2017 & & & & & & \\
M0 & 280.6 & 7.9 & 26.694 & 1 & 49.068 & 56.127 \\
Mt & 274.6 & 6.9 & 0.00 & 0 & 24.375 & 34.963 \\
Mb & 257.9 & 3.3 & 0.00 & 0 & 24.375 & 34.963 \\
2018 & & & & & & \\
MO & 279.4 & 4.0 & 18.121 & 1 & 49.170 & 47.360 \\
Mt & 277.3 & 3.6 & 0.00 & 0 & 24.049 & 34.833 \\
Mb & 271.6 & 2.0 & 0.00 & 0 & 24.049 & 34.833 \\
\hline
\end{tabular}

AIC: criterio de información de Akaike; BIC: criterio de información bayesiano; DM: desviación del modelo; GL: grados de libertad; NR: casos estimados en R; SE: error estándar.

Fuente: elaboración propia en base a la estimación utilizando la librería estadística Rcapture.

\section{Tabla 3}

Subregistro y exhaustividad del Sistema Epidemiológico de Tuberculosis (SIEPI-TB) y Sistema de Información Gerencial de Tuberculosis (SIGTB), a en la Región Cajamarca, Perú, años 2017 y 2018.

\begin{tabular}{cccccccc}
\hline Año & Casos & \multirow{2}{*}{ IC95\% } & \multicolumn{2}{c}{ Subregistro (\%) } & \multicolumn{3}{c}{ Exhaustividad (\%) } \\
& estimados & & SIGTB & SIEPI-TB & SIGTB & SIEPI-TB & $\begin{array}{c}\text { SIGTB y } \\
\text { SIEPI-TB }\end{array}$ \\
\hline 2017 & 275 & $262.40-289.43$ & 40.7 & 20.4 & 59.3 & 79.6 & 91.6 \\
2018 & 277 & $270.98-284.85$ & 25.6 & 11.6 & 74.4 & 88.4 & 97.1 \\
\hline
\end{tabular}

IC95\%: intervalo de 95\% de confianza.

Fuente: elaboración propia en base a casos estimados y a casos registrados en cada sistema de vigilancia. 
Tabla 4

Características epidemiológicas de los casos de tuberculosis (TB) registrados en el Sistema Epidemiológico de Tuberculosis (SIEPI-TB) de la Región Cajamarca, Perú, años 2017 y 2018.

\begin{tabular}{|c|c|c|}
\hline Característica & Frecuencia (n) & $\%$ \\
\hline \multicolumn{3}{|l|}{ Sexo } \\
\hline Masculino & 294 & 63,4 \\
\hline Femenino & 170 & 36,6 \\
\hline \multicolumn{3}{|l|}{ Seguro de salud } \\
\hline Seguro Integral de Salud (SIS) & 374 & 80,6 \\
\hline Seguro Social de Salud (EsSalud) & 60 & 12,9 \\
\hline Seguro de las fuerzas armadas & 2 & 0,4 \\
\hline Seguros privados & 1 & 0,2 \\
\hline No cuenta con seguro de salud & 27 & 5,8 \\
\hline \multicolumn{3}{|l|}{ Criterio de diagnóstico } \\
\hline Bacteriológico & 273 & 58,8 \\
\hline Radiológico & 88 & 19,0 \\
\hline Histopatológico & 50 & 10,8 \\
\hline Clínico & 39 & 8,4 \\
\hline Clínico epidemiológico & 6 & 1,3 \\
\hline Otros & 8 & 1,7 \\
\hline \multicolumn{3}{|l|}{ Localización } \\
\hline Pulmonar & 349 & 75,2 \\
\hline Pleural & 36 & 7,8 \\
\hline Meníngea & 19 & 4,1 \\
\hline Miliar & 13 & 2,8 \\
\hline Ganglionar & 13 & 2,8 \\
\hline Genitourinaria & 8 & 1,7 \\
\hline Piel & 3 & 0,6 \\
\hline Gastrointestinal & 3 & 0,6 \\
\hline Renal & 3 & 0,6 \\
\hline Ósea & 2 & 0,4 \\
\hline Otras & 15 & 3,2 \\
\hline \multicolumn{3}{|l|}{ Perfil de resistencia } \\
\hline Sensible & 452 & 97,4 \\
\hline Monorresistencia a isoniacida & 5 & 1,1 \\
\hline Monorresistencia a rifampicina & 3 & 0,6 \\
\hline TB MDR & 4 & 0,9 \\
\hline \multicolumn{3}{|l|}{ Comorbidades y factores } \\
\hline Alcoholismo & 32 & 6,9 \\
\hline Drogadicción & 20 & 4,3 \\
\hline Diabetes & 21 & 4,5 \\
\hline VIH-SIDA & 19 & 4,1 \\
\hline \multicolumn{3}{|l|}{ Condición de egreso } \\
\hline Sin condición de egreso & 267 & 57,5 \\
\hline Curados & 124 & 26,7 \\
\hline Fallecidos & 39 & 8,4 \\
\hline Abandono & 18 & 3,9 \\
\hline Tratamiento completo & 8 & 1,7 \\
\hline No iniciaron tratamiento & 6 & 1,3 \\
\hline Fracaso & 1 & 0,2 \\
\hline Excluido por mal diagnóstico & 1 & 0,2 \\
\hline
\end{tabular}

Nota: resultados combinados de dos años (total de casos: 464).

Fuente: base de datos deduplicados de SIEPI-TB. 
En cuanto a características epidemiológicas en la Región Cajamarca, el sexo masculino fue el más afectado, coincidiendo con otros investigadores 11,19, siendo predominante la TB pulmonar, diagnosticada por criterio bacteriológico. Estos datos concuerdan con la información nacional y de países limítrofes 14,20; acerca del aseguramiento en salud la mayoría de personas afectadas con TB estuvo afiliada al Seguro Integral de Salud (SIS), esto, debido a que el SIS es un seguro público en Perú y presenta mayor cobertura, además está destinado a personas con bajos recursos en condición de pobreza, situación vinculada estrechamente a la TB 19; en cuanto al promedio de edad de personas afectadas con esta enfermedad, estos corresponden a población económicamente activa, coincidiendo con otros autores 3,16 , lo cual tiene un importante impacto socioeconómico en la región.

El mayor porcentaje de casos correspondió a TB sensible, valor similar a los datos a nivel nacional 21; si bien es cierto el Perú se ubica dentro de los 30 países con más alta carga de TB MDR 1, en la Región Cajamarca un bajo porcentaje de casos corresponde a esta forma, debido a que la mayoría de casos de TB MDR del Perú se concentran en Lima y Callao 21; por otra parte se encontró que el primer factor asociado a la tuberculosis en esta región fue el alcoholismo, seguido del consumo de drogas; existe evidencia de la relación de estos factores con la tuberculosis 22,23,24, acerca de las frecuencias obtenidas para las comorbilidades TB-VIH; TB-diabetes, los valores encontrados concuerdan con los reportados a nivel nacional 3 .

Respecto de la condición de egreso al tratamiento, se encontró bajo porcentaje de fracaso terapéutico; también se evidenció un bajo porcentaje de casos que egresaron como curados, datos que difieren drásticamente de lo encontrado en la literatura, donde se reporta porcentajes exitosos de personas con alta terapéutica en condición de curadas 25,26. Esto sugiere una preocupante y seria deficiencia en la actualización de los sistemas por parte de las unidades notificantes, pues a la fecha de estudio se encontró un elevado porcentaje sin condición de egreso, por otra parte el abandono al tratamiento debe ser considerado un punto importante a ser vigilado por las autoridades sanitarias de la región.

Una posible limitación del estudio se relaciona con el uso de datos secundarios, los cuales son alimentados por diversas unidades notificantes existentes en todas las redes de salud de la región, indicamos también que el error de registro y falta de información específica limita ampliar el análisis de algunas variables.

Los datos permiten concluir que en la Región Cajamarca existe un preocupante subregistro de casos en los sistemas de vigilancia sanitaria de tuberculosis, existiendo la necesidad del monitoreo de los mismos, constituyendo un problema de salud pública que debe ser evaluado por las autoridades sanitarias; el método de captura recaptura permite determinar el subregistro de tuberculosis y la evaluación confiable de los sistemas de vigilancia de tuberculosis, así como un acercamiento a la tasa de incidencia real de tuberculosis en la región. 


\section{Colaboradores}

H. R. Quiroz-Ruiz participó en la elaboración del diseño del proyecto, análisis estadístico de datos y redacción del artículo. J. L. Sosa-Flores participó en la redacción y en la revisión crítica del contenido del artículo. F. N. Hernández-Palomino colaboró en la revisión de datos y redacción del artículo.

\section{Conflictos de interés}

Los autores informan la inexistencia de cualquier tipo de conflicto de intereses.

\section{Informaciones adicionales}

ORCID: Hans Ramón Quiroz-Ruiz (0000-00028482-8328); Jorge Luis Sosa-Flores (0000-00024095-8139); Fiorella Nathalí Hernández-Palomino (0000-0001-7474-9717).

\section{Agradecimientos}

A la Universidad Nacional Pedro Ruiz Gallo (UNPRG) y a la Dirección Regional de Salud Cajamarca (DIRESA Cajamarca) por la autorización para desarrollar este estudio.

\section{Referencias}

1. World Health Organization. Global tuberculosis report 2019. Geneva: World Health Organization; 2019.

2. Organización Panamericana de la Salud. Tuberculosis en las Américas 2018. Washington DC: Organización Panamericana de la Salud; 2018.

3. Alarcón V, Alarcón E, Figueroa C, MendozaTicona A. Tuberculosis en el Perú: situación epidemiológica, avances y desafíos para su control. Rev Peru Med Exp Salud Pública 2017; 34:299-310.

4. Sanghavi D, Gilman R, Lescano-Guevara A, Checkley W, Cabrera L, Cardenas V. Hyperendemic pulmonary tuberculosis in a Peruvian shantytown. Am J Epidemiol 1998; 148:384-9.

5. Culqui DR, Trujillo OV, Cueva N, Aylas R, Salaverry O, Bonilla C. Tuberculosis en la población indígena del Perú 2008. Rev Perú Med Exp Salud Pública 2010; 27:8-15.

6. Gobierno del Perú. Ley no 30.287 de Prevención y Control de la Tuberculosis en el Perú. Esta ley tiene el objeto de regular los mecanismos de articulación entre los sectores involucrados en la prevención y el control de la tuberculosis, garantizando la cobertura y continuidad de una política de Estado de lucha contra esa enfermedad. Diario Oficial El Peruano 2014; 14 dic.

7. King R, McCrea RS. Capture-recapture methods and models: estimating population size. Handbook of Statistics 2019; 40:33-83.

8. Rivest L-P, Baillargeon S. Loglinear models for capture-recapture in R. http://www2. uaem.mx/r-mirror/web/packages/Rcapture/ vignettes/RcaptureJSS.pdf (accedido el Sep/ 2020).

9. Rivest L-P, Baillargeon S. Rcapture: loglinear models for capture-recapture experiments. https://cran.r-project.org/web/packages/ rcapture/rcapture.pdf (accedido el Sep/2020).

10. Posada SL, Rosero-Noguera R. Comparison of mathematical models: an application for evaluation of animal food. Rev Colomb Cienc Pecu 2007; 20:141-8.

11. Múñiz-González F, Guerra-Laso J, GarcíaGarcía S, López-Veloso M, Raposo-García S, Carracedo-Falagán N, et al. Aproximación a la incidencia real de tuberculosis en el Área de Salud de León: aplicación del método capturarecaptura para comparar 2 fuentes de información. Enferm Infecc Microbiol Clín 2013; 31:82-7.

12. World Health Organization. Global tuberculosis report 2017. Geneva: World Health Organization; 2017.

13. Hernández-Sarmiento JM, Andrade H, Murillo LE, Velásquez L, Gómez-Restrepo AJ, Robledo J. Mortalidad por tuberculosis, estudio del registro en municipios del departamento del Chocó, Colombia, 2012. Enferm Infecc Microbiol 2018; 38:38-43. 
14. Ospina S. La tuberculosis, una perspectiva histórico-epidemiológica. Infectio 2001; 5:24150.

15. Li T, Shewade HD, Soe KT, Rainey JJ, Zhang H, $\mathrm{Du} \mathrm{X}$, et al. Under-reporting of diagnosed tuberculosis to the national surveillance system in China: an inventory study in nine counties in 2015. BMJ Open 2019; 9:e21529.

16. Iglesias-Gozalo MJ, Rabanaque-Hernández MJ, Gómez López LI. La tuberculosis en la provincia de Zaragoza. Estimación mediante el método captura-recaptura. Rev Clin Esp 2002; 202:249-54.

17. Tejero Encinas S, Asensio Villahoz P, Vaquero Puerta JL. Vigilancia epidemiológica de la tuberculosis pulmonar atendida en el nivel especializado a partir de dos fuentes de información: Valladolid. Rev Esp Salud Pública 2003; 77:211-20.

18. Dunbar R, Van Hest R, Lawrence K, Verver S, Enarson D, Lombard C, et al. Capture-recapture to estimate completeness of tuberculosis surveillance in two communities in South Africa. Int J Tuberc Lung Dis 2011; 15:1038-43.

19. Bermejo MC, Clavera I, Michel de la Rosa FJ, Marín B. Epidemiología de la tuberculosis. An Sist Sanit Navar 2007; 30 Suppl 2:7-19.

20. Flores N. La tuberculosis un problema multicausal y es tarea de todos ponerle fin. Boletín Epidemiológico del Perú 2019; 28:244-5.
21. Mendoza-Ticona A, Moore DA, Alarcón V, Samalvides F, Seas C. Propuesta de esquemas de tratamiento antituberculosis basados en la susceptibilidad a isoniacida y rifampicina. Rev Perú Med Exp Salud Pública 2013; 30:197-204.

22. Lönnroth K, Williams BG, Stadlin S, Jaramillo E, Dye C. et al. Alcohol use as a risk factor for tuberculosis - a systematic review. BMC Public Health 2008; 8:289.

23. Rehm J, Samokhvalov AV, Neuman MG, Room $\mathrm{R}$, Parry C, Lönnroth K. The association between alcohol use, alcohol use disorders and tuberculosis (TB): a systematic review. BMC Public Health 2009; 9:450.

24. Ramos Zambrano KE, Silva Peralta JP, Piedrahita Icaza AS, Toaza Suarez VC. La drogadicción como uno de los agentes desencadenantes de la tuberculosis. Revista Científica de Investigación Actualización del Mundo de las Ciencias 2019; 3:60-7.

25. Escobar SN. Situación epidemiológica de la tuberculosis en Chile en el escenario global 2018. Rev Chil Enferm Respir 2019; 35:63-70.

26. Ascarza JC. Baciloscopía al inicio del tratamiento anti-tuberculoso como factor pronóstico de la condición de egreso de pacientes con tuberculosis pulmonar tratados en el Hospital Cayetano Heredia en el período 1973-2012 [Tesis de Graduación]. Lima: Universidad Peruana Cayetano Heredia; 2018. 


\section{Abstract}

This study sought to determine the underreporting of tuberculosis (TB) cases in the Cajamarca Region, Peru in 2017 and 2018 through the capturerecapture method and to estimate the TB incidence rate using this method, assess the exhaustiveness of surveillance systems, and describe the epidemiological characteristics of TB in this period. A descriptive, retrospective study was performed in the Cajamarca Region; the surveillance systems were analyzed were the TB Management Information System (SIGTB) and the TB Epidemiological System (SIEPI-TB). Based on the number of cases recorded in each system, the capture-recapture method was applied to obtain an estimation of real cases, validating the data with a log-linear model in the $R$ statistical environment. The largest underreporting rates in the systems were $40.7 \%$ in 2017 and 25.6\% in 2018; the estimated incidence rates in both years were higher than those reported by the official systems, the most exhaustive surveillance system was SIEPI-TB. The results indicate the existence of worrisome underreporting and the need to monitor the TB surveillance systems.

Tuberculosis; Underregistration; Health Surveillance

\section{Resumo}

Esta pesquisa procurou aferir a subnotificação de casos de tuberculose (TB) na Região de Cajamarca, nos anos 2017 e 2018 usando o método de captura-recaptura, também para estimar a ta$x a$ de incidência através deste método, avaliar a abrangência dos sistemas de vigilância e descrever as características epidemiológicas da tuberculose no período estudado. Um estudo descritivo e retrospectivo foi realizado na Região de Cajamarca; foram analisados dois sistemas de vigilância: o Sistema de Informação de Gestão da Tuberculose (SIGTB) e o Sistema Epidemiológico da Tuberculose (SIEPI-TB). Com base no número de casos registrados em cada sistema, o método de captura-recaptura foi aplicado para obter uma estimativa de casos reais, validando os dados com um modelo log-linear no ambiente estatístico $R$. As maiores subnotificações nos sistemas foram: $40,7 \% \mathrm{em}$ 2017 e 25,6\% em 2018; verificou-se que as taxas de incidência estimadas em ambos os anos foram mais altas do que as relatadas pelos sistemas oficiais, e que o sistema de vigilância mais abrangente foi o SIEPI-TB. Os resultados indicam a existência de uma subnotificação preocupante e a necessidade de monitoramento dos sistemas de vigilância da TB.

Tuberculosis; Sub-Registro; Vigilância Sanitária
Recibido el 19/Sep/2020

Versión final presentada el 09/Nov/2020

Aprobado el 13/Nov/2020 